\title{
Stability of an Extemporaneously Compounded Oral Suspension of Bosentan
}

\author{
Alina Malik, PharmD*; Gregory Gorman, $P h D^{\dagger}$; Lori Coward, BS ; and John J. Arnold, PhD
}

\begin{abstract}
Purpose: To assess the stability of an extemporaneously compounded oral suspension of bosentan from commercially available tablets for a period of 1 month.

Methods: A $6.25 \mathrm{mg} / \mathrm{mL}$ oral suspension of bosentan monohydrate was prepared from Tracleer tablets. The bosentan suspension was then evenly divided between 2 light-resistant prescription bottles and stored in the dark either under refrigeration $\left(4-8^{\circ} \mathrm{C}\right)$ or at controlled room temperature $\left(21-26^{\circ} \mathrm{C}\right)$. The suspensions were assessed for physical changes (ease of resuspendability, change in color, change in odor), and samples were drawn immediately after preparation and on days 0,1 , $3,7,10,14,21,28$, and 31 . Samples were analyzed at each time point by high-performance liquid chromatography (HPLC) utilizing a reversed-phase column with chemical stability defined as the retention of at least $90 \%$ of the initial intact bosentan concentration measured.

Results: No change in suspendability, color, or odor of the compounded bosentan suspensions was noted throughout the storage period. Furthermore, regardless of storage conditions, the oral suspension of bosentan retained at least $94 \%$ of the active pharmaceutical ingredient for 31 days after preparation.

Conclusion: The results of our study indicate that a $6.25 \mathrm{mg} / \mathrm{mL}$ bosentan oral suspension stored in the dark under refrigeration and at room temperature maintains physical and chemical stability for 1 month.
\end{abstract}

Key Words—bosentan, compounded, pulmonary arterial hypertension, stability, suspension

Hosp Pharm—2016;51:389-395

$\mathrm{P}$ ulmonary arterial hypertension $(\mathrm{PAH})$ is a progressive illness that occurs as the pulmonary arteries and capillaries in the lungs begin to narrow and become blocked due to the proliferation of various cells, in particular smooth muscle cells and endothelial cells, within this location. This narrowing or blockage results in increased pulmonary artery pressure and pulmonary vascular resistance, as the lower right ventricle of the heart must pump more forcefully to move blood through the lungs. In severe cases, the heart muscle may fail as a result of increased contractility due to the narrowed or blocked arteries and capillaries. ${ }^{1}$ One possible facilitator of PAH is endothelin-1, an endogenous vasoconstrictor and smooth muscle mitogen. Endothelin-1 is often overproduced in the plasma and lung tissue of patients who suffer from PAH. Two endothelin-1 receptors, known as type $\mathrm{A}$ endothelin $\left(\mathrm{ET}_{\mathrm{A}}\right)$ and type $B$ endothelin $\left(\mathrm{ET}_{\mathrm{B}}\right)$, are prevalent in the lungs and vascular tissue. ${ }^{1,2}$ Bosentan monohydrate (Figure 1), marketed as Tracleer, is an orally active agent that has demonstrated efficacy for the treatment of pulmonary arterial hypertension (PAH). ${ }^{1,3,4}$ Bosentan is a substituted pyrimidine derivative that functions as an antagonist at both receptor types, although it has a slightly greater affinity for $\mathrm{ET}_{\mathrm{A}}$ receptors. ${ }^{5}$ Bosentan has been shown to decrease remodeling of the pulmonary vasculature, which is one of the main complications associated with PAH. This remodeling occurs as the blood vessel walls thicken in response to increased smooth muscle cell production and decreased cell apoptosis. Bosentan also decreases the

${ }^{*}$ Pharmacy Student, ${ }^{\dagger}$ Associate Professor of Pharmaceutical Sciences, ${ }^{\star}$ Research Associate, ${ }^{\S}$ Associate Professor of Pharmaceutics, McWhorter School of Pharmacy, Samford University, Birmingham, Alabama. Corresponding author: John J. Arnold, PhD, McWhorter School of Pharmacy, Samford University, 800 Lakeshore Drive, Birmingham, AL 35229; phone: 205-726-4040; fax: 205-726-2088; e-mail: jarnold@samford.edu 
<smiles>COc1ccccc1Oc1c(NS(=O)(=O)c2ccc(C(C)(C)C)cc2)nc(-c2ncccn2)nc1OCCO</smiles>

Figure 1. Chemical structure of bosentan.

rate of clinical worsening in patients with advanced $\mathrm{PAH}$ and improves exercise ability. ${ }^{1,2}$

Bosentan has been extensively studied for the treatment of PAH in pediatric populations. ${ }^{6-12}$ In several studies, a bosentan dosage of $2 \mathrm{mg} / \mathrm{kg}$ per dose has been shown to be efficacious in children with PAH, with higher dosages unlikely to provide any additional benefits. ${ }^{6,9}$ Unfortunately, in the United States, bosentan is currently only supplied in tablet form in strengths of either $62.5 \mathrm{mg}$ or $125 \mathrm{mg} .{ }^{5}$ Consequently, administration of bosentan in children can be challenging, because reduced and more individualized dosages are often required. This results in the need to prepare an extemporaneously compounded liquid formulation. To our knowledge, no uniform preparation guidelines with validated beyond use dating for a compounded bosentan liquid preparation currently exist. The purpose of this study is to prepare an extemporaneously compounded bosentan suspension using commercially available compounding supplies and evaluate its stability under controlled room temperature and at refrigeration over the course of 1 month.

\section{METHODS}

\section{Preparation of Bosentan Oral Suspension}

An important consideration in the preparation of bosentan oral suspension is that, according to the National Institute for Occupational Safety and Health (NIOSH), bosentan is a hazardous medication and should be handled accordingly. ${ }^{13}$ Consequently, certain safety measures should be taken in the preparation of the bosentan oral suspension as dictated by NIOSH, as well as the United States Pharmacopeia (USP) $<795>$ chapter entitled Pharmaceutical Compounding - Nonsterile Preparations. ${ }^{13,14}$ These safety measures are intended to protect the health and welfare of the compounder by ensuring that only personnel trained in handling hazardous medications and using certain personal protective equipment (PPE) engage in such compounding. Briefly, PPE can include double gloving, wearing a protective gown, and using a respiratory mask if preparation does not occur in a fume hood.

Three $62.5 \mathrm{mg}$ tablets of bosentan monohydrate (Actelion Pharmaceuticals US, Inc, lot number: HP015P0101; expiration date: March 2018) were triturated in a porcelain mortar and pestle. A small amount of glycerin (Actelion Pharmaceuticals US, Inc., lot number: HP015P0101; expiration date: March 2018) was added to the mortar to aid in the trituration of the tablets to a fine powder. Next, a 1:1 preparation of the commercially available pharmacy compounding vehicles, FlavorPlus (Humco, lot number: 544980; expiration date: October 2016) and FlavorSweet (Humco, lot number: 545314; expiration date: January 2017), were produced in a separate container. A portion $(10 \mathrm{~mL})$ of the FlavorPlus:FlavorSweet mixture was added to the mortar and vigorously triturated. Subsequently, another $10 \mathrm{~mL}$ of the FlavorPlus:FlavorSweet mixture was then added to the mortar with trituration. Afterwards, the mixture was transferred to a 2-oz lightresistant prescription bottle. Finally, the mortar was rinsed with $10 \mathrm{~mL}$ of FlavorPlus:FlavorSweet, which was then added to the prescription bottle to bring the final volume up to $30 \mathrm{~mL}$ and achieve a $6.25 \mathrm{mg} / \mathrm{mL}$ concentration. The suspension was mixed well to ensure homogeneity and then divided equally between 2 light-resistant prescription bottles so that one bottle could be stored at refrigerated temperature $\left(4-8^{\circ} \mathrm{C}\right)$ and one bottle could be stored at controlled room temperature $\left(21-26^{\circ} \mathrm{C}\right)$. Both the refrigerated and room temperature bottles were stored in the dark. Following resuspension by shaking, 3 samples of $0.6 \mathrm{~mL}$ each were extracted from both the room temperature and refrigerated bottles on days $0,1,3,7,10,14,21,28$, and 31 . These samples were stored at $-80^{\circ} \mathrm{C}$ until analysis via high-performance liquid chromatography (HPLC) could be performed.

\section{Sample Preparation and HPLC Analysis}

Stability samples from this study were assayed using a previously published HPLC stabilityindicating assay for bosentan monohydrate that was validated per ICH guidelines. ${ }^{15}$ Sample analysis was conducted on a Shimadzu (Columbia, MD) LC-20AD HPLC with a SPD-M20 photodiode array detector and SIL-20 autosampler. Briefly, this method, which is capable of separating up to 5 impurities observed from various forced degradation conditions, utilizes a Zorbax SB-Phenyl $(250 \mathrm{~mm} \times 4.6 \mathrm{~mm}, 5 \mu \mathrm{m})$ column (Phenomenex, Torrance CA) maintained at $35^{\circ} \mathrm{C}$. A gradient elution profile at a flow rate of $1 \mathrm{~mL} / \mathrm{min}$ consisted of a mixture of $40 \%$ methanol and $60 \%$ phosphate buffer $(10 \mathrm{mM})$ with $0.1 \%$ triethylamine, 
adjusted to $\mathrm{pH} 2.5$ with orthophosphoric acid, used as solvent $\mathrm{A}$ and acetonitrile was used as solvent B. The gradient profile started at 70/30 (A:B) for 5 minutes and was then increased in a linear profile to $40 / 60$ over 25 minutes. It was held for 5 minutes before returning to $70 / 30$ for a 5 -minute re-equilibration. A photodiode array detector set at $220 \mathrm{~nm}$ was used to collect sample data from $10 \mu \mathrm{L}$ injections.

A stock solution of bosentan monohydrate (1 $\mathrm{mg} / \mathrm{mL}$ ) was prepared by dissolving the bulk drug (Cayman Chemical, Ann Arbor MI, lot number: 0455131-2; expiration date: October 2017) in methanol. Calibration standards over a range of 15.6 to $156.3 \mu \mathrm{g} / \mathrm{mL}$ were prepared from the stock solutions using methanol as a diluent and assayed as described above. Preparation of stability samples consisted of diluting a $200 \mu \mathrm{L}$ portion of the formulation to $1: 100$ with methanol followed by thorough vortex mixing and sonification as required to affect complete dissolution. A $1 \mathrm{~mL}$ aliquot of the diluted formulation was placed in an autosampler vial for analysis. The residual concentration of bosentan was measured in the stability samples and reported as percent of the day 0 concentration.

\section{RESULTS}

\section{Method Verification}

A freshly prepared standard of bosentan $(62.5 \mathrm{~g} /$ $\mathrm{mL}$ ) was analyzed via the HPLC method described, and the chromatogram is presented in Figure 2. To ensure accurate quantitation of the parent compound using the chromatography from the stability-indicating assay, calibration standards were assayed to verify the linear range of the method as used in this study. Analysis of replicate bosentan calibration standards over the range of 15.6 to $156.3 \mu \mathrm{g} / \mathrm{mL}$ on 3 separate days resulted in a linear response with $r^{2}>0.999$ on each day of analysis (Figure 3). The percent relative

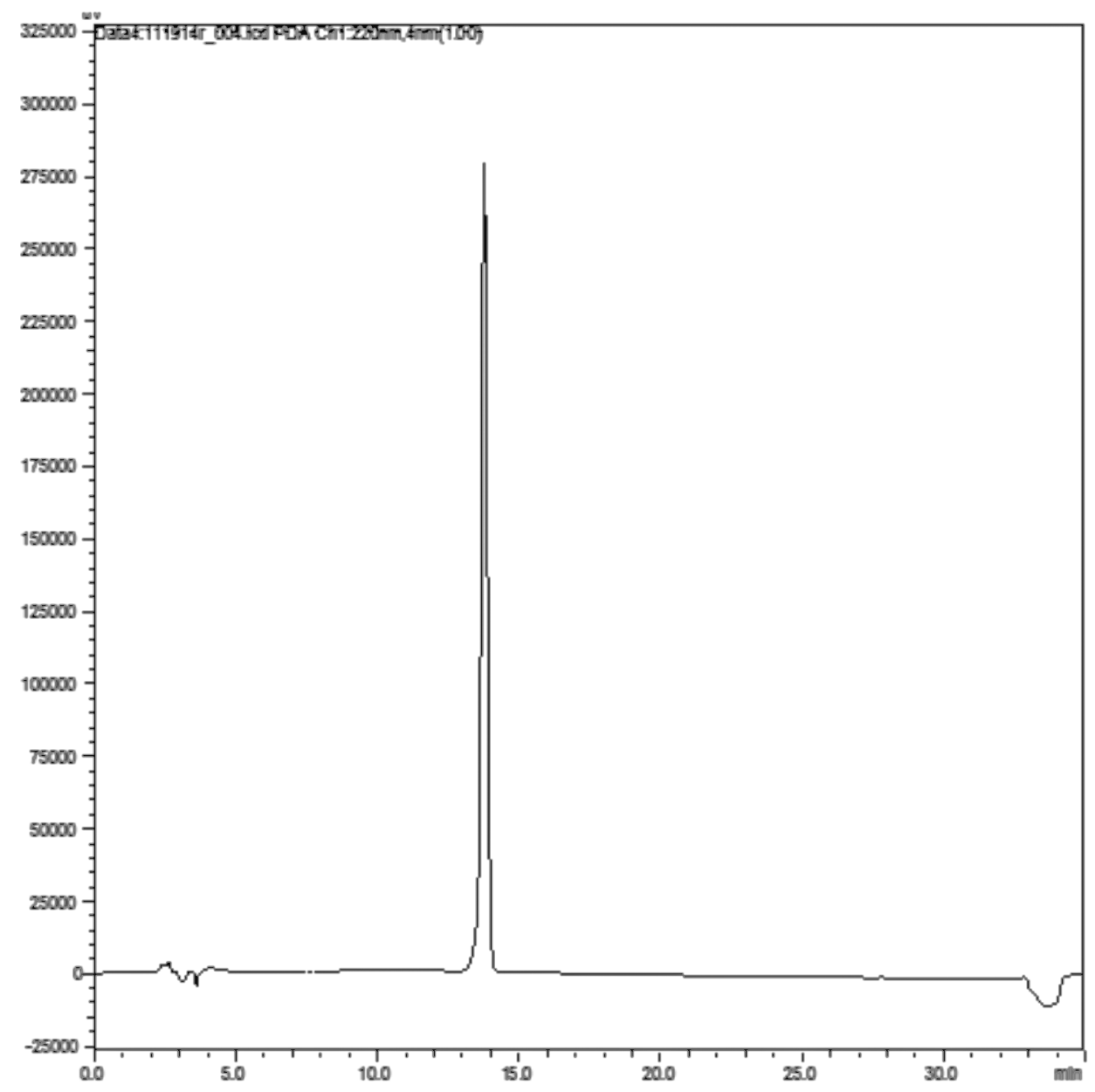

Figure 2. High-performance liquid chromatogram of bosentan standard $(62.5 \mathrm{mg} / \mathrm{mL})$. 
standard deviation ( $\%$ RSD) ranged from $1.5 \%$ to $2.7 \%$ at $15.6 \mu \mathrm{g} / \mathrm{mL}$ and from $0.36 \%$ to $3.3 \%$ at 156 $\mu \mathrm{g} / \mathrm{mL}$ over the 3 separate days. The overall \%RSD was less than $3.5 \%$ for all 3 days. To demonstrate the

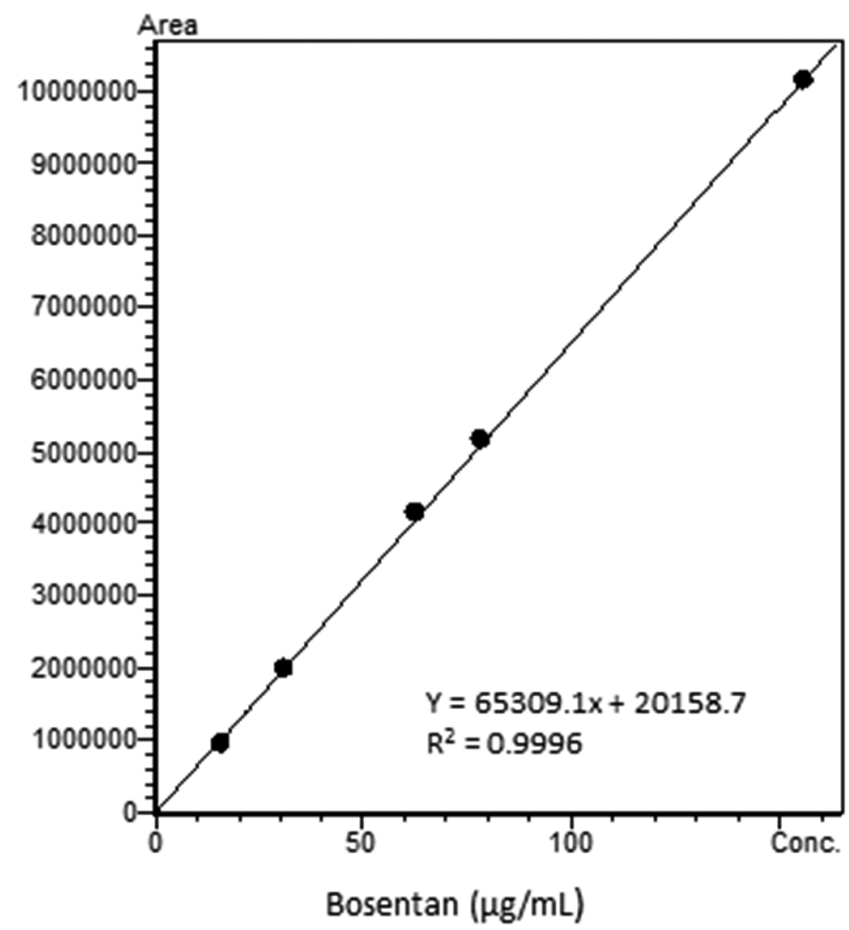

Figure 3. Bosentan calibration curve from 15.6 to $156.3 \mu \mathrm{g} / \mathrm{mL}$. ability of the assay to chromatographically resolve degradents, bosentan was subjected to acidic, alkaline, and oxidative stress conditions. Acidic and alkaline conditions consisted of heating bosentan at $60^{\circ} \mathrm{C}$ in either $12 \mathrm{~N} \mathrm{HCl}$ or $5 \mathrm{~N} \mathrm{NaOH}$ for 2 and 3.5 hours, respectively. Oxidative degradents were generated by exposing bosentan to a $3 \% \mathrm{H}_{2} \mathrm{O}_{2}$ solution at room temperature for 24 hours. Figure 4 shows the overlayed chromatograms demonstrating the assays' ability to resolve degradents from intact bosentan.

\section{Formulation Stability}

When prepared as described, the resultant compounded bosentan oral suspension $(6.25 \mathrm{mg} / \mathrm{mL})$ was pharmaceutically elegant with excellent suspendability. Whether stored at controlled room temperature or under refrigeration, the compounded suspensions were observed to maintain their physical stability throughout the duration of their storage and were able to be easily resuspended upon shaking at each sampling time point. Neither the odor nor the color of the compounded suspensions changed throughout the period of study, suggesting a lack of bacterial growth in the compounded preparations. Samples from both the room temperature and refrigerated suspensions were collected at the prescribed time points and analyzed via HPLC to ensure that bosentan remained chemically stable throughout the study period. No

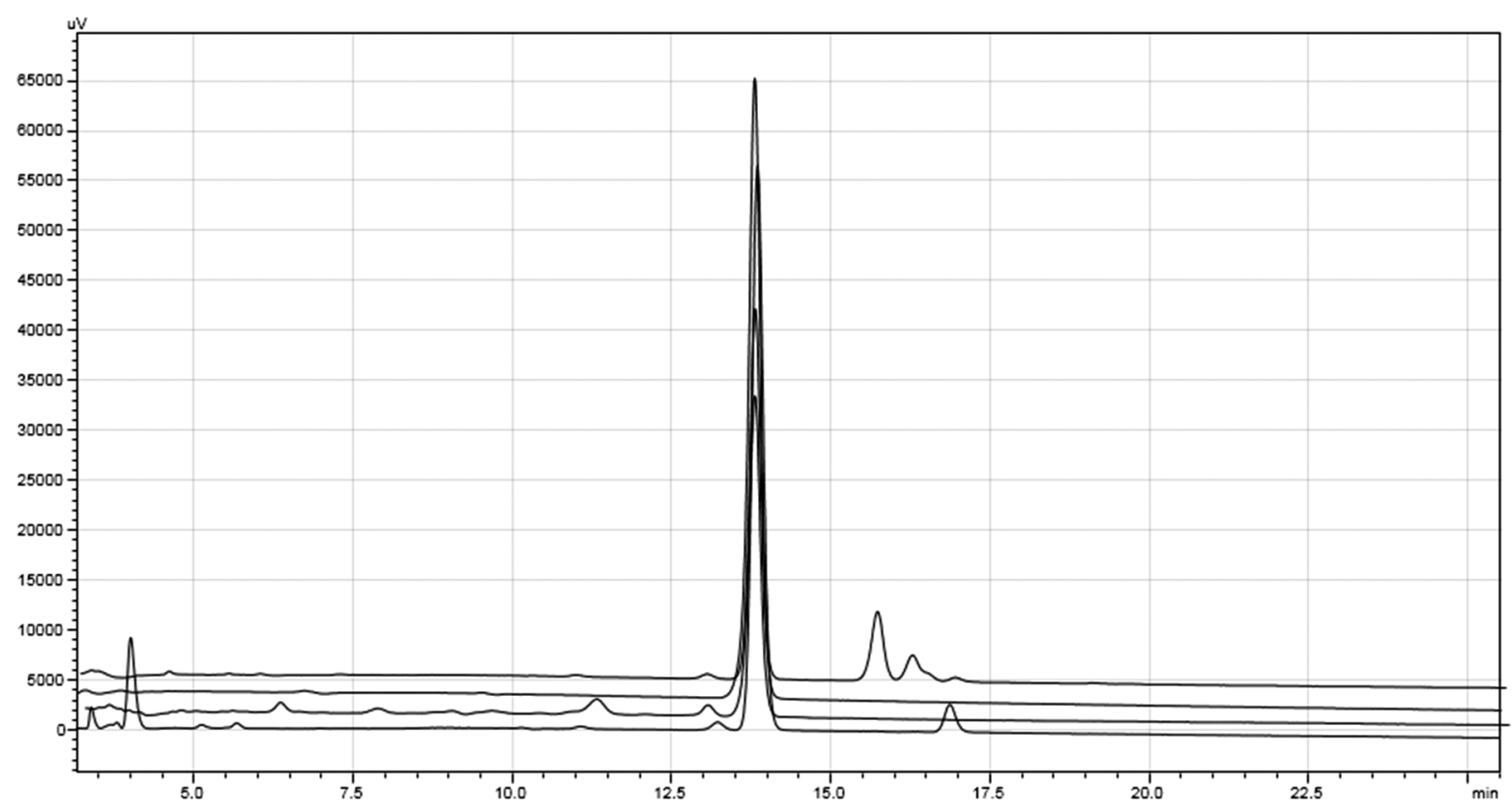

Figure 4. Overlayed high-performance liquid chromatograms from forced degradation study from top to bottom: acid, base, hydrogen peroxide, bosentan standard. 
substantial degradation of bosentan was found to have occurred in samples from either formulation based on the measured concentration of bosentan as a percent of the original formulation concentration. Additionally, no peaks in the chromatograms corresponding to decomposition products were observed at either storage condition at 31 days (Figure 5). All assayed time point samples maintained acceptable potency (at least $90 \%$ of the starting concentration) at each time point throughout the study period (Table 1). At 31 days, the suspension stored under refrigeration was almost $96 \%$ intact and the suspension stored under room temperature was almost $94 \%$ intact.

\section{DISCUSSION}

Bosentan monohydrate (Tracleer) is an important drug for the treatment of PAH due to its plethora of beneficial effects. ${ }^{3,4,6-12}$ Bosentan has become a significant drug in the treatment of $\mathrm{PAH}$ in pediatric patients; however, the doses required for treatment in this patient population are often not achievable with available tablet formulations of the marketed preparation..$^{6-12}$ Consequently, this often leaves practitioners with no choice but to compound liquid preparations of bosentan with no validated beyond use date. In the current study, we have tested a simple method of preparation of a bosentan oral suspension

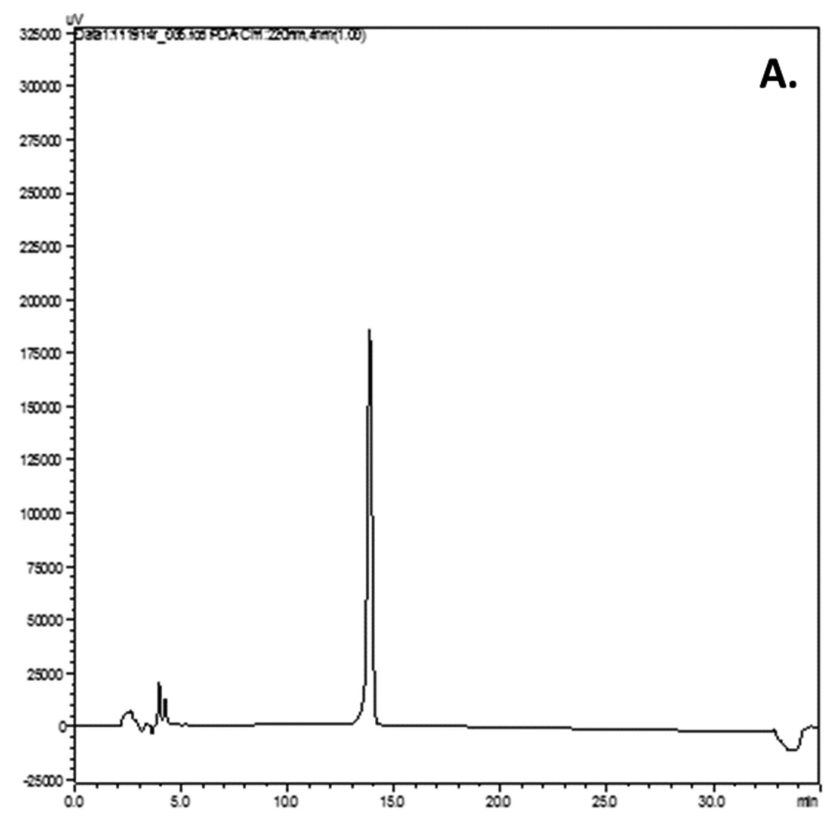

using commercially available compounding supplies. Furthermore, we have assessed the stability of this preparation in order to assign a valid beyond use date.

A $6.25 \mathrm{mg} / \mathrm{mL}$ oral suspension of bosentan was prepared using FlavorPlus and FlavorSweet, both of which are pharmacy compounding vehicles commercially available from Humco. The method of preparation was relatively simple, requiring only a mortar and pestle, and resulted in an elegant pharmaceutical suspension. The oral suspensions, whether stored at controlled room or refrigerated temperatures, remained pharmaceutically elegant with easy resuspendability at each time point throughout the course of the study. In terms of retarding bacterial growth, both of the compounding vehicles contain the pharmaceutical preservatives methylparaben and potassium sorbate. There was no change in odor or color of the compounded bosentan suspensions throughout the course of the study, which indicates a lack of bacterial growth. Consequently, we concluded that compounded bosentan suspensions are physically stable when prepared by the method presented.

The chemical stability of the $6.25 \mathrm{mg} / \mathrm{mL}$ oral suspension of bosentan was assessed at controlled room and refrigerated temperatures. After 1 month, both preparations retained a content uniformity of at least $90 \%$ of their initial concentration (Table 1).

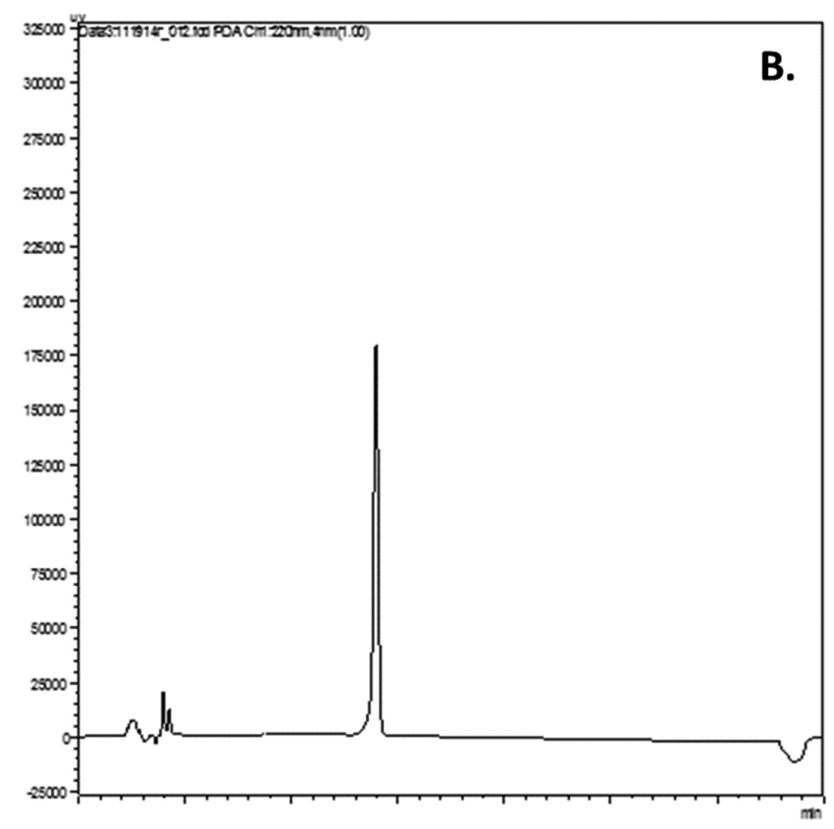

Figure 5. High-performance liquid chromatograms of bosentan oral suspensions at 31 days after storage at $(A) 4^{\circ}$ to $8^{\circ} \mathrm{C}$ or (B) $21^{\circ}$ to $26^{\circ} \mathrm{C}$. 
Table 1. Percent of initial concentration remaining of bosentan measured in oral suspension over a period of 31 days as determined by HPLC analysis

\begin{tabular}{ccc}
\hline \multirow{2}{*}{ Storage time, days } & \multicolumn{2}{c}{$\%$ of initial concentration } \\
\cline { 2 - 3 } & $4^{\circ} \mathrm{C}$ to $8^{\circ} \mathrm{C}$ & $21^{\circ} \mathrm{C}$ to $26^{\circ} \mathrm{C}$ \\
\hline 0 & $100^{\mathrm{a}}$ & $100^{\mathrm{a}}$ \\
\hline 1 & $95.2 \pm 3.3$ & $101 \pm 2.2$ \\
\hline 3 & $93.7 \pm 2.8$ & $98.7 \pm 0.6$ \\
\hline 5 & $99.7 \pm 6.5$ & $93.2 \pm 0.4$ \\
\hline 7 & $94.8 \pm 1.8$ & $114 \pm 12$ \\
\hline 10 & $99.0 \pm 1.6$ & $95.5 \pm 1.4$ \\
\hline 14 & $95.7 \pm 1.6$ & $101 \pm 0.4$ \\
\hline 21 & $98.0 \pm 0.9$ & $95.8 \pm 2.3$ \\
\hline 28 & $96.7 \pm 0.9$ & $98.7 \pm 0.8$ \\
\hline 31 & $95.9 \pm 0.8$ & $93.7 \pm 0.7$ \\
\hline
\end{tabular}

Note: Samples were assayed in triplicate and data represent mean \pm standard error of the mean.

${ }^{a}$ Initial concentration $5.64 \pm 0.51 \mathrm{mg} / \mathrm{mL}$.

Additionally, no degradation products of bosentan were detected in any of the samples from either of the storage conditions according to the HPLC analysis (Figure 4). Therefore, we concluded that the bosentan suspensions are chemically stable when prepared by the method presented and stored at either room temperature or under refrigerated conditions. Our conclusion from this study is further supported by data that suggest that bosentan is an inherently stable molecule. In forced degradation studies under moderate stress conditions $\left(0.1 \mathrm{~N}\right.$ acid or base at $80^{\circ} \mathrm{C}$ for 30 minutes), less than $2.5 \%$ loss of bosentan was observed, while losses due to thermal, oxidative, and photolytic stress conditions were less than $2 \%{ }^{16}$ In a separate study under extreme stress conditions (12 N HCl or $5 \mathrm{~N} \mathrm{NaOH}$ refluxed for 4 or 48 hours, respectively), losses of less than $15 \%$ were observed..$^{15}$ It is important to note that care should be taken in preparing these solutions for dispensing and that patients should be consulted on the proper handling and storage of the compounded product per USP $<795>$ guidelines on compounding nonsterile products. ${ }^{14}$ The beyond use date should be clearly indicated on the container, while storage conditions and signs of instability (eg, changes in color, odor, or resuspendability) should be clearly and thoroughly conveyed to the patient.

\section{CONCLUSION}

Based on the results obtained from this study, it can be concluded that bosentan maintains its physical and chemical integrity when compounded into a $6.25 \mathrm{mg} / \mathrm{mL}$ oral suspension with the commercially available pharmacy vehicles FlavorPlus and FlavorSweet. For those practitioners using bosentan to treat pediatric patients with $\mathrm{PAH}$, these results validate that compounded oral suspensions of bosentan can be used for up to 1 month when stored under controlled room or refrigerated temperatures.

\section{ACKNOWLEDGMENTS}

The authors wish to thank Dr. Chesca Barnett from the Department of Pharmacy at Children's of Alabama for generously supplying the bosentan monohydrate (Tracleer) used in this study.

\section{REFERENCES}

1. Gabbay E, Fraser J, McNeil K. Review of bosentan in the management of pulmonary arterial hypertension. Vasc Health Risk Manag. 2006;41:254-259.

2. Humbert M, Barst RJ, Robbins IM, et al. Cellular and molecular pathobiology of pulmonary arterial hypertension. J Am Coll Cardiol. 2004;43:13S-24S.

3. Rubin LJ, Badesch DB, Barst RJ, et al. Bosentan therapy for pulmonary arterial hypertension. $N$ Engl J Med. 2002;346:896-903.

4. McLaughlin VV, Sitbon O, Badesch DB, et al. Survival with first line bosentan in pulmonary arterial hypertension. Eur Respir J. 2005;25:244-249.

5. Bosentan. In: Clinical Pharmacology. Tampa, FL: Gold Standard. Updated April 29, 2013. http://clinicalpharmacologyip.com.ezproxy.samford.edu/Forms/Monograph/monograph.asp $\mathrm{x}$ ?cpnum=2336\&sec=mondesc\&t=0. Accessed May 15, 2015.

6. Barst RJ, Ivy D, Dingemanse J, et al. Pharmacokinetics, safety, and efficacy of bosentan in pediatric patients with pulmonary arterial hypertension. Clin Pharmacol Ther. 2003;73:372-382.

7. Rosenzweig EB, Ivy DD, Widlitz A, et al. Effects of longterm bosentan in children with pulmonary arterial hypertension. J Am Coll Cardiol. 2005;46:697-704.

8. Maiya S, Hislop AA, Flynn Y, Haworth SG. Response to bosentan in children with pulmonary hypertension. Heart. 2006;92:664-670.

9. Beghetti M, Haworth SG, Bonnet D, et al. Pharmacokinetic and clinical profile of a novel formulation of bosentan in children with pulmonary arterial hypertension: The FUTURE-1 study. Br J Clin Pharmacol. 2009;68:948-955. 
10. Beghetti M. Bosentan in pediatric patients with pulmonary arterial hypertension. Curr Vasc Pharmacol. 2009;7:225-233.

11. Carter NJ, Keating GM. Bosentan: In pediatric patients with pulmonary arterial hypertension. Paediatr Drugs. 2010;12:63-73.

12. Ivy DD, Rosenzweig EB, Lemarie J-C, et al. Long-term outcomes in children with pulmonary arterial hypertension treated with bosentan in real-world clinical settings. Am J Cardiol. 2010;106:1332-1338.

13. NIOSH list of antineoplastic and other hazardous drugs in healthcare settings. 2014. http://www.cdc.gov/niosh/docs/2014138/pdfs/2014-138_v3.pdf. Accessed February 11, 2016.
14. USP <795> Pharmaceutical Compounding-Nonsterile Preparations. 2014. http://www.usp.org/sites/default/files/ usp_pdf/EN/gc795.pdf. Accessed February 11, 2016.

15. Jadhav SA, Shashikant LB, Jadhav SL, et al. Stabilityindicating gradient RP-LC method for the determination of process and degradation impurities in bosentan monohydrate: An endothelin receptor antagonist. Chromat Res Int. 2011;1-9. 16. Annapurna MM, Pavani S, Anusha S, Harika M. Validated stability indicating RP-HPLC method for the determination of bosentan in presence of degration products. Indo Am J Pharm Res. 2013;3:4528-4540. 